\title{
Steganography in Audio Files by Entropy using FEC as Reed-Solomon of VOIP Streams
}

\author{
P.S. Santhana Mangai \\ PG Student, IT Dept, SNS College of Technology \\ Sathy Road, Coimbatore
}

\author{
G.Selva Vinayagam \\ Assistant professor, IT Dept, SNS College of Tech \\ Sathy Road, Coimbatore
}

\begin{abstract}
In this paper introduce a novel technique to identify the voice (active frames) and silent regions (inactive frames) of a speech stream very much suitable for VoIP calls. Thus here the proposed a better voice activity detection based on the entropy algorithm. High-capacity steganography algorithm for embedding data in the inactive frames. Then inactive frames are encoded by G.723.1 source codec, which is used extensively in Voice over Internet Protocol (VoIP).As the data embedding capacity is very high on inactive frames of the audio signals than in the active frames. Entropy based Voice Activity Detection algorithms for VoIP applications can save bandwidth by filtering the frames that do not contain speech .On evaluating the proposed approach with the existing methods, our approach yield a better saving in bandwidth, yet maintaining high capacity of data embedding. yet maintaining good quality of the speech streams and then finally using forward error correcting code as Reed-Solomon codes. It can be used as encoder and decoder. By using Reed-Solomon code, data losses occur in the transmission can be detected and recovered by adding extra information (redundancy) to the original data.
\end{abstract}

\section{Key words}

Audio streams, Inactive frames, Entropy, Steganography, Voice over Internet Protocol (VoIP), Reed-Solomon.

\section{INTRODUCTION}

STREAMING media, such as Voice over Internet Protocol (VoIP) streams, are broadcast live over the Internet and delivered to end-users. Security remains one of the main challenges with this new technology. With the upsurge of VoIP applications available for use in recent years, VoIP streams become one of the most interesting cover objects for modern steganography. Digital steganography in low bit rate audio streams is commonly regarded as a challenging topic in the field of data hiding. There have been several steganography methods of embedding data in audio streams. For example, Wu et al. [1] sug-gested a G.711-based adaptive speech information hiding approach.Aoki [2] proposed a technique of lossless steganography in G.711 encoded speeches. Ma et al. [3] framed a steganography method of embedding data in G.721 encoded speeches. All these methods adopt high bit rate audio streams encoded by the waveform codec as cover objects, in which plenty of least significant bits exist. Long distance calls are expensive while transported over Public Switched Telephone Networks (PSTN). Thus the current trend is to provide this service on data networks, especially in the light of popular applications like Skype, Yahoo, etc. IP suite, originally built for data traffic, works on the best effort delivery principle. Since resource sharing through statistical multiplexing is used in data networks, the total number of calls supported may be enhanced at the gateways, which bridge PSTN to the IP networks through speech compression techniques or codec's like G.722.1 [4], iLBC [5], or GSM [6]. Therefore such services can be economical compared to circuit-switched networks for long distance calls.

However, data network does not guarantee a faithful voice transmission and reproduction as in PSTN. We need to keep the packet delay, loss and delay jitter under check. One of the simple ways to reduce the delay at the playout buffer is to detect the talk spurts and transmit only those segments. This, while reducing the bandwidth, also avoids building up of play out buffer. Thus there is a need for applying Voice Activity Detection (VAD) algorithms to detect the talk spurts for a voice call on the Internet which is the central theme of this paper. Bandwidth saving with VAD can be independent of the codec's used. We also note that VAD algorithm should be as simple as possible so that it can be implemented on many simple portable devices in real-time. Conversational speech is a sequence of contiguous segments of pauses and speech bursts [7]. The strategy is to use the fact that no speech channel is continuously active. In a conversational setup, typically contribution from each party is less than $50 \%$ of the time [8]; Kaleed et al., report a $40 \%$ activity of speech in VoIP. In fact even when one party is speaking, there are times when sizeable pauses between words and expressions exist [9]. Thus VAD algorithms take recourse to speech pattern classification to differentiate between speech and silence (pause) periods to save bandwidth.

A speech segment may be classified as an active or an inactive (silent or non-speech), based on its energy. The term inactive segment refers to a period of incomprehensible sound which may not have zero-energy [10].

REED-SOLOMON (RS) codes are non-binary linear block Codes with a wide range of applications in wireless communications and storage systems.

RS codes concatenated with convolution codes are the standard channel codes for satellite transmission and deepspace communications. They are also outer codes in the third generation (3G) wireless standard and expected to be used as Outer codes in concatenated coding schemes for fourth generation wireless standard.

RS codes are maximum distance separable which is a very attractive property for error-correcting codes. Hard decision decoders with low complexity exist for RS codes [11], but they don't use the channel reliability information which causes considerable performance loss that may not be acceptable in many applications. 


\section{RELATED WORK}

The analysis by synthesis (ABS)-based speech information approach was adopted to embed speech data in an original speech carrier, with good efficiency in steganography and good quality of output speech [12]. Recently, linear predictive Coefficients were substituted with secret speech data by using an ABS speech coding scheme [13], but the experimental results available are very limited. Krätzer, Dittmann, and Vogel [14] argued that the inactive voice of a speech was not suitable for a being used as a cover object for steganography owing to an obvious distortion of the original speech.

By contrast, Huang et al. [15] suggested an algorithm for embedding information in some parameters of the speech frame encoded by ITU G.723.1 codec, without leading to distinction between inactive voices and active voices. These are computationally complex and require training and building a model. Entropy measure is employed in many of the speech recognition solutions. Waheed et al. use entropy for speech segmentation based on Shen's work [12]. Generic reedSolomon encoder [13].Linear-algebraic list decoding of folded Reed-Solomon codes [14]

\section{PRINCIPLE OF STEGANOGRAPHY IN INACTIVE AUDIO FRAMES}

\subsection{Hangover Algorithm for Detecting Active Voices}

The Entropy based VAD is used to decide whether the current audio frame is an active voice by comparing the energy of the frame (Enr) with a threshold (Thr).VAD $=0$ means the frame is an inactive voice; otherwise, the frame is an active voice.

\subsection{Energy of a Frame}

The energy of a frame indicates possible presence of speech Information and is a useful parameter for VAD algorithms. Let $s(n)$ is the $n$th sample of speech. If the length of the frame were $N$ samples, then the energy for the $j$ th frame can be represented as

$$
E(j)=\begin{aligned}
& \mathrm{N}-1 \\
& n=0
\end{aligned} s^{2}(n)
$$

where $j=1 ;::: ; N f$ and $N f$ is the number of frames for a speech stream.

\section{PARAMETERS FOR VAD DESIGN}

\subsection{Choice of Frame Duration}

Active frames bundled together are transmitted and queued up in a packet-buffer at the receiver. Consider, a VoIP system having buffer of 7-10 packet. Having a packet size equivalent to $10 \mathrm{~ms}$ allows the VoIP system to start playing the audio at the receiver's end after $30-40 \mathrm{~ms}$ from the time the queue start building up.

If the frame duration were $50 \mathrm{~ms}$, an initial delay would be of $150-200 \mathrm{~ms}$, which is unsuitable since, maximum round trip delay within $400 \mathrm{~ms}$ [15] for a good quality speech. Therefore, the frame duration must be chosen properly. VoIP system may use 5-60 ms frame sizes and we see many popular VoIP applications use $60 \mathrm{~ms}$ packet size. The speech is assume to quasi-stationary for $20 \mathrm{Ms}$. Thereby the spectral entropy measure is also assumed to be reliable and hence validity of decision.
Thus use in our algorithm $20 \mathrm{~ms}$ speech frames. A packet may contain many of these frames depending on $t$ design of the application.

\subsection{Entropy Based VAD algorithm for VOIP}

VAD was used first in Speech Recognition systems, Compression and Speech coding [16]-[17] to find the beginning and ending of talk spurts. For VoIP applications stringent detection of beginning and ending of talk spurts is not needed. Coding techniques like [18] use built-in VAD but they are computationally expensive. Sovka and Pollak have reported their work using spectral subtraction [19] and cepstrum [20], [21], mainly for speech enhancement systems.

Entropy measure is employed in many of the speech recognition solutions. Waheed et al. use entropy for speech segmentation based on Shen's work [12]. Interestingly, both of them use this method for the recorded speech samples to effectively filter the speech bursts, so that later these bursts can be used to recognize the uttered speech.

They use overlapping frames with each frame of approximately 25 ms length with a $25-50 \%$ overlap. Also they construct a histogram with a varying number of bins in the range of 50 to 100. The entropy is calculated and compared with a fixed threshold which is slightly above the midpoint of maximum and minimum entropy values considered. This calls for screening the whole recorded speech file. In this paper a threshold based on variable median, tracking the actual entropy for each frame in real-time is used. The measure is same as in [12],[22], however our approach is considerably different while adopting it for real-time frame by frame decision. Also we take into account the varying nature of speech characteristics vis-'a-vis background noise, as well as speaker independent methodology. We tune entropy feature to suit the VoIP voice packets.

\subsection{Desirable Aspects of VAD Algorithms}

The list some desirable aspects of good VAD algorithms for VoIP applications.

- VAD algorithm must implement a good decision rule that exploits the properties of speech to consistently classify segments of speech into inactive and active.

- It should adapt to non-stationary background noise to

- Enhances robustness.

- The computational complexity of VAD algorithm must be low to suit real-time applications.

- Toll quality voice should be achieved after applying VAD algorithm.

- VAD must maximize the detection of inactive periods to save the bandwidth.

The assumptions on the VAD algorithm proposed here are based on the following characteristics

- Speech is quasi-stationary. Its spectral form changes over short periods, e.g. 20-30 ms.

- Background noise is relatively stationary, changing very slowly with time.

- Energy of the speech signal is usually higher than background noise energy; else speech will be unintelligible.

\section{EMBEDDING ALGORITHM}

The embedding process is divided into four steps:-

Step 1) Voice activity detection. The speech with PCM format is divided into frames be an active voice; otherwise, the frame 
is marked with "S". Each frame is inputted into the VAD detector that adopts the residual energy algorithm.

Step 2) Encoding all frames by G.723.1 codec. Regardless of the frame type, all the frames, and, are encoded by using the standard G.723.1 algorithm with $6.3 \mathrm{~kb} / \mathrm{s}$. The resulting low bit rate audio stream containing active and inactive frames is then outputted from the codec.

Step 3) Embedding information in frames. According to the frame type, two different steganography algorithms are used to embed information in the frames. The algorithm 1 is used to embed the secret information in the inactive frame. The expression denotes the algorithm 2 is used to embed the information in the active frame.

Step 4) Encapsulation and sending. The inactive frames and active frames with hidden information are encapsulated in VoIP packets, which are transmitted over the Internet [23].

\section{REED-SOLOMON WORK}

REED-SOLOMON (RS) codes are non-binary linear block codes with a wide range of applications in wireless communications and storage systems.

\subsection{Reed-Solomon Encode}

Reed Solomon (RS) codes are a mechanism to detect and correct burst of errors in data transmission and storage systems. It provides a solid introduction to foundation mathematical concept of Galois Field algebra and its application. Reed Solomon (RS) codes are block-based error-correcting mechanism in a wide range of applications in digital transmission and storage.

The RS encoder takes a block of data and adds extra redundant bits. A block of RS codes described in Figure 1 consists of $K$ information symbols added by $2 t$ parity symbols to make an $N$ symbol codeword. In this scheme, the RS decoder can correct up to $t$ symbols that contain errors in the codeword, and specified as $R S(N, K)[13]$

This section gives an overview of RS encoding process with a short data as an example, i.e. RS $(7,3)$. It means that the RS codes have seven codeword symbols, three of which are the original information symbols and the rest are parity symbols. Let each is 3-bit symbol. The information that will be transmitted is divided into three 3-bit blocks, each of which is added by four 3-bit symbols to make 7 code words symbols. The field elements for $m=3$ using primitive polynomial $x 3+x$ +1 are presented in Table 1 .

A Reed Solomon codeword is constructed using a special polynomial called generator polynomial.

Thus, a Reed Solomon encoding process consists of the following steps:
1. Multiply the information symbols with $X(N-K)$. This can be done by shifting the information symbols to the left to allow space for $2 t$ parity symbols.

2. Divide the result of step 1 with the generator polynomial using GF algebra

3. Add the result of step 2 (remainder of division) to the result of step $1[13]$

\begin{tabular}{|c|l|lll|}
\hline Elemen & Polynomial & $\alpha$ & $\alpha$ & $\alpha$ \\
2 & 1 & 0 \\
\hline 0 & 0 & 0 & 0 & 0 \\
\hline$\alpha^{0}$ & 1 & 0 & 0 & 1 \\
\hline$\alpha^{1}$ & $\alpha$ & 0 & 1 & 0 \\
\hline$\alpha^{2}$ & $\alpha^{2}$ & 1 & 0 & 0 \\
\hline$\alpha^{3}$ & $\alpha+1$ & 0 & 1 & 1 \\
\hline$\alpha^{4}$ & $\alpha(\alpha+1)=\alpha^{2}+\alpha$ & 1 & 1 & 0 \\
\hline$\alpha^{5}$ & $\alpha^{3+\alpha^{2}=\alpha^{2}+\alpha+}$ & 1 & 1 & 1 \\
\hline$\alpha^{6}$ & 1 & 1 & 0 & 1 \\
\hline
\end{tabular}

Table 1: Elements of GF $\left(2^{3}\right)$

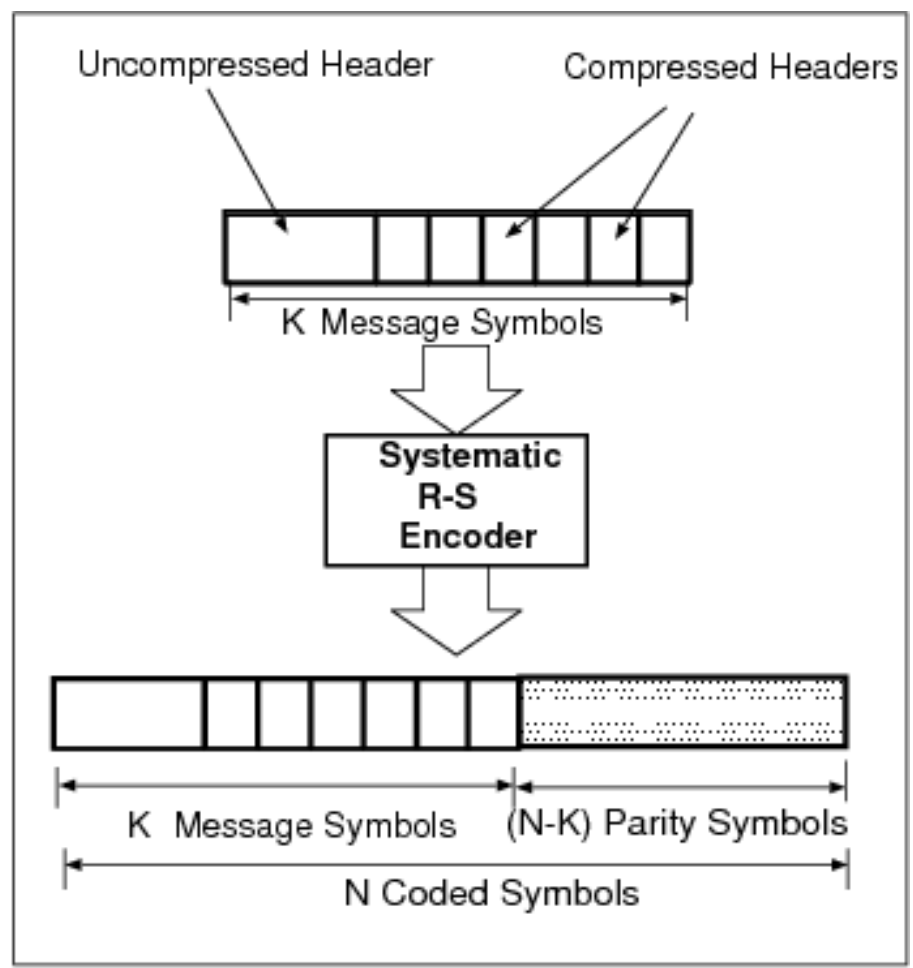

Figure 1: Reed-Solomon Encoder

\subsection{Reed-Solomon Decode}

1. The first step in decoding a Reed-Solomon code is to calculate the $2 \mathrm{t}$ syndrome components $\mathbf{S}(\mathrm{X})$.

2. The second step in decoding a Reed-Solomon code is to find the error location polynomial $\mathbf{L}(\mathrm{X})$ and the error evaluation polynomial $\mathbf{W}(\mathrm{X})$. 
3. The last step in decoding a Reed-Solomon code is to find the error location and the error value.[24]

\section{EXTRACTING ALGORITHM}

The extraction of secret information from the stegospeech is the inverse process of the embedding algorithm, and it is divided into the following three steps.

Step 1) Receiving and decapsulation. The VoIP packets, are received, buffered, and then decapsulated by the receiver.

Step 2) Decoding and active frame detection. The buffered frames are copied to the decoding buffer and decoded into the PCM formatted audio stream. The improved VAD method is then used to distinguish between inactive frames and active frames.

Step 3) Extracting secret information. The inactive and active frames of the low bit rate audio stream are identified. The secret information is then extracted from by using Algorithms 1 and 2. Algorithms 1 and 2 are used to extract the secret information from the inactive frames and the active frames respectively [23].

\section{CONCLUSION}

The proposed a novel algorithm for VAD using spectral entropy based measure to find active and inactive zones in ga speech stream.In this paper, we have suggested a high-capacity steganography algorithm for embedding data in the inactive frames of low bit rate audio streams encoded by G.723.1 source codec. The experimental results have shown that our proposed steganography algorithm can achieve a larger data embedding capacity with imperceptible distortion of the original speech, compared with other algorithms. RS algorithm comes into practical use in covert VoIP communications, it is necessary to explore how to assure the integrity of hidden messages in the case of packet loss, security with a focus of securely transferring the data without making packet loss has to be occurred.

\section{REFERENCES}

[1] Z.Wu andW. Yang, "G.711-based adaptive speech information hiding approach," Lecture Notes Comput. Sci., vol. 4113, pp. 1139-1144, 2006.

[2] N. Aoki, "A technique of lossless steganography for G.711 telephony speech," in Proc. 2008 4th Int. Conf. Intelligent Inf. HidingMultimedia Signal Process. (IIH-MSP), Harbin, Aug. 2008, pp. 608-611.

[3] L. Ma, Z. Wu, and W. Yang, "Approach to hide secret speech information in G.721 scheme," Lecture Notes Comput. Sci., vol. 4681, pp. 1315-1324, 2007.

[4] "Low-complexity coding at 24 and $32 \mathrm{kbit} / \mathrm{s}$ for hands-free operation in systems with low frame loss," International Telecommunication Union, Recommendation G.722, 2001.

[5] S. Andersen, A. Duric, H. Astrom, R. Hagen, W. Kleijn, and J. Linden,"Internet low bit rate codec (iLBC)," Internet Engineering Task Force,RFC 3951, 2001.

[6] "GSM-enhanced full rate specifications," European Telecommunications Standards Institute, Specification 06.51, 06.60-63 and 06.82, 2001

.[7] B. Gold and N. Morgan, Speech and Audio Signal Processing. New York: John Wiley and Sons, 2000.
[8] J. Natvig, S. Hansen, and J. De Brito, "Speech processing in the paneuropean digital mobile radio system (GSM) system overview," in Proc. IEEE Global Telecommunications Conference (IEEE GLOBECOM 1989), vol. 2, Nov. 1989, pp. 1060-1064.

[9] K. El-Maleh and P. Kabal, "Natural quality background noise coding using residual substitution," in Proc. EUROSPEECH, vol. 5, Sept. 1999, pp. 2359-2362.[7] A. M. Kondoz, Digital Speech. New York: John Wiley and Sons, 1999.

[10] R. V. Prasad, A. Sangwan, H. S. Jamadagni, and M. C. Chiranth, "Comparison of voice activity detection algorithms for voip," in Proc.IEEE Symposium on Computer and Communications, vol. 5, July 2002,pp. 530-535.

[11] S. Lin and D. Costello, Error Control Coding. PrenticeHall, 1983.

[12] Z.Wu,W. Yang, and Y. Yang, "ABS-based speech information hiding approach," Electron. Lett., vol. 39, no. 22, pp. 1617-1619, Oct. 2003.

[13] Petrus Mursanto," GENERIC REED SOLOMON ENCODER", Fakultas Ilmu Komputer, Universitas Indonesia, Depok 16424, Indonesia.

[14] VENKATESAN GURUSWAMI," Linear-algebraic list decoding of folded Reed-Solomon codes", Computer Science Department Carnegie Mellon University Pittsburgh, PA 15213,2011.

[15] Voice Activity Detection for VoIP-An Information Theoretic Approach. R. V. Prasadø, R. Muralishankary, Vijay S.z, H. N. Shankary, Przemysław Pawełczakø and Ignas Niemegeers, PES Institute of Technology.

[16] C. Krätzer, J. Dittmann, T. Vogel, and R. Hillert, "Design and evaluation of steganography for voice-over-ip," in Proc. IEEE Int. Symp. Circuits Syst., May 2006, pp. 2397-3234.

[17] C. Bao, Y. F. Huang, and C. Zhu, "Steganalysis of compressed speech," in Proc. IMACS Multiconf. Computational Eng. Syst. Applicat.(CESA), Oct. 2006, pp. 5-10.

[18] "Coding of speech at $8 \mathrm{kbit} / \mathrm{s}$ using conjugate-structure algebraic-codeexcitedlinear-prediction (CS-ACELP)," International Telecommunication Union, Recommendation G.729, 1996.

[20] P. Pollak, P. Sovka, and J. Uhlir, "The noise suppression system for a car," in Proc. EUROSPEECH, vol. 5, Sept. 1993, pp. 1073-1076.

[22] K. Waheed, K. Weaver, and F. M. Salam, "A robust algorithm for detecting speech segments using an entropic contrast," in Proc. 45 ${ }^{\text {th }}$ IEEE International Midwest Symposium on Circuits and Systems, vol. 3, Aug. 2002, pp. 328-331. 